\title{
An Ultrasound Based Biomedical System for Continuous Cardiopulmonary Monitoring: A Single Sensor for Multiple Information
}

\author{
Amirhossein Shahshahani, Zeljko Zilic and Sharmistha Bhadra.
}

\begin{abstract}
Biomedical wearable sensors enable long-term monitoring applications and provide instantaneous diagnostic capabilities. Physiological monitoring can help in both the diagnosis and the ongoing treatment of a vast number of cardiovascular and pulmonary diseases such as hypertension, dysrhythmia, and asthma. In this paper, we present a system capable of monitoring several vital signals and physiological variables that determine the cardiopulmonary activity status. We explore direct measurements of multiple vital parameters with only one sensor and without special constraints. The system employs a PZT4 piezo transducer stimulated by a suitable analog front-end. The system both generates pulsed ultrasound waves at $1 \mathrm{MHz}$ and amplifies reflected echoes to track internal organ motions, mainly that of the heart apex. According to the respiratory motion of the heart, the proposed system provides respiratory and heart cycles information. Promising results were obtained from six subjects with an average accuracy of $96.7 \%$ in heartbeats per minute (BPM) measurement, referenced to a commercial photoplethysmography sensor. It also exhibits $94.5 \%$ sensitivity and $94.0 \%$ specificity in respiration detection compared to a SPR-BTA spirometer signal as a reference.
\end{abstract}

Index Terms-Cardiopulmonary monitoring, Heart rate variability (HRV), Piezo transducer, Respiratory sinus arrhythmia (RSA), Ultrasound.

1

\section{INTRODUCTION}

Health monitoring systems may contain many types of sensors, leading the world of Internet of Things (IoT) [1]. Non-invasive monitoring of living organs with high resolution is essential for observing physiological activity, mainly the breathing and heart rates as well as their patterns. Besides the respiratory rate, depth and patterns are important. The obstructive sleep apnoea (OSA) is the most common form of such diseases in which breathing is interrupted by a blockage of airflow. Correct detection of OSA also requires heart rate variability analysis in addition to monitoring respiratory rate and pattern [2].

${ }^{1}$ Manuscript received April 11, 2018; revised Nov 19, 2018; accepted April 8, 2019. Date of publication zzzz XX, YYYY; This research was funded by McGill university [G130100 Start Up Fund Electrical and Computer Engineering] and Natural Sciences and Engineering Research Council of Canada [G245366 NSERC RGPIN-2018-05176]. (Corresponding author: Amirhossein Shahshahani.)

A. Shahshahani, Z. Zilic and S. Bhadra are with the Department of Electrical and Computer Engineering, McGill university, Montreal, Canada. (Email: amirhossein.shahshahani@mail.mcgill.ca).

Copyright (c) 2017 IEEE. Personal use of this material is permitted. However, permission to use this material for any other purposes must be obtained from the IEEE by sending an email to pubs-permissions@ieee.org.
Numerous non-invasive devices for respiration monitoring are proposed. Appropriate use of current monitoring systems and correct assessment on the provided data are essentials in accurate and trustable diagnosis. Systems based on direct method are flow meters that use pressure transducers or even thermal and ultrasound flow meters [3], [4]. Aluminum nitride piezoelectric films [5] [6] can measure and monitor pressure fluctuations applied on the sensor due to respiration and heartbeat on the contact surface when a subject is lying on it. A similar concept is measuring beats and elongation of the abdomen or chest circumference using fiber-optic sensor [7]. Another example of a direct method is chest and abdomen movements analysis using accelerometer sensors [8]. Since these methods are based on the heart beats felt on the skin surface or chest and abdomen circumference changes due to the breathing, they are sensitive to human motions. A contactless breathing rate monitoring by low frequency ultrasound sensor is proposed by S. D. Min et al [9]. It presents the ultrasonic proximity sensor approach by detecting the time of flight of ultrasound waves between the transmitted and received signal during respiration in the abdominal wall-motion. Accordingly, it can hardly be applied on different situations and positions, especially when the patient is dressed. A better solution is proposed by P. Arlotto et al [10] by monitoring airflow from nose or mouth based on the frequency shift in ultrasound waves (Doppler effect).

Indirect methods are based on a biofeedback of an organ activity. As an example, pulse oximetry (PPG) sensors measure oxygen level variations due to the breathing [11] [12] as well as small blood pressure variations that the pulmonary system applies. They are dependent on many parameters - for instance, the oxygen level depends on oxygen density of the area the person is in [13], bad functions of lungs or airflow, latency between the breathing and oxygen level changes in blood, uncertainty in breath detection at fast respirations and many relevant parameters [11]. In addition, it is not favorable to carry a sensor on the finger, earlobe or forehead [14].

Foremost cardio-respiratory monitoring methods are based on pulse oximetry sensor (PPG) [15], ECG signal, skin conductivity [16]. ECG signal monitoring devices require at least two conductive electrodes being mounted on chest skin. ECG and PPG devices are the most accurate and conventional methods in hospitals, specifically for critically ill patients. PPG sensors as the most popular and cheap method for heart rate monitoring are not applicable for the direct respiratory monitoring method, as discussed before and will be explained more 
in section IV. Skin conductivity assessment (plethysmography) provides a way to measure respiratory activity by measuring the thoracic impedance changes caused by inspiration and expiration. Accordingly, this method requires at least two conductive electrodes which makes it uncomfortable as a wearable or long time monitoring system. Moreover, the movementinduced artifact on its signal cannot be easily removed by filtering methods [17]. Similar technique is investigated to measure heart rate [18].

Many conventional techniques are not practical in all conditions such as when the patient is dressed or under different body position or motion. As a health monitoring system, it is more advantageous if the device monitors more than one feature while operating under different body positions with less constraints on hardware resources and wiring connectivities. In this paper we report an ultrasound based organ motion tracking system to extract cardiopulmonary information such as respiration patterns, heart rate and its variation (HRV).

Capabilities of ultrasound systems in tracking the inner organ motions and blood flows, wall motion abnormality in heart [19] and some other information such as cardiac output measurements [20] were studied using medical ultrasound machines, such as an echocardiogram. They are expensive and can not be used as a wearable device. In this study, we focus on applicability of ultrasound sensor as a wearable device for health monitoring. The proposed system operates the same way as devices such as echocardiogram machine. However, this system has potential to be utilized as a low cost wearable device. We employed only one transducer in this study while the probe of medical ultrasound machines contains at least 50 transducers. In addition, this sensor is capable of monitoring the respiratory signal. The reported power consumption of this work is negligible compared to the existing ultrasound machines in the market.

An efficient and direct method to address the need on monitoring both heart and respiration cycles is monitoring the heart motions. Circumference of the normal adult rib cage expands by 3 to $5 \mathrm{~cm}$ during inhalation [21]. Moreover, respiratory motion of the heart on some patients in [22] and [23] shows that during inspiration, the heart moved inferiorly and underwent a craniodorsal rotation, and in few patients, they observed anterior movements as well. The amount of motions in [22] were $4.9 \pm 1.9 \mathrm{~mm}$, on average for the caudally translation, and for some subjects $1.3 \pm 1.8 \mathrm{~mm}$ on average for the anterior translation. On the other hand, the external intercostal muscles are most significant in respiration, having fibers that are angled obliquely downward and forward from rib to rib [24]. The contraction of these fibers raises each rib toward the rib above, with the overall effect of raising the rib cage, assisting in inhalation. Considering all motions due to the respiration, there is a possibility to extract heart and respiratory waveforms from ultrasound reflections from the heart and surrounding tissues.

In this study, the point of interest to be tracked is the heart apex. The human chest contains twelve ribs, the first 7 referred to as true ribs, as they are attached to the sternum directly. The heart is situated in the middle mediastinum, at the level of the thoracic vertebrae of ribs T5-T8 [25]. Although the heart is behind the rib cage, its motions can be seen by ultrasound from intercostal spaces. Hence, a piezo sensor placed in either intercostal between T5 and T8 can detect heart and surrounded internal organs motions.

Ultrasound technology (US) as an inexpensive, safe, and real-time capable can be a direct method for cardiopulmonary monitoring. Ultrasound imaging systems with an array of piezo transducers is widely used in diagnosis and imaging. In this study we used only one sensory node consisting of a PZT-4 piezo transducer as a 1-D trackers of internal organs. A mixed signal embedded system is designed. The system uses a sensory node, operating in B-mode which plots ultrasound echoes as a function of intensity and time (depth) as an image. In this mode, the system generates ultrasound pulses discontinuously. By recording the intensity and time of flight (ToF) of reflected echoes from observing organs, the position of the organ can be found. To determine the velocity and motion of the organ over time, the system records the amplitude and depth of specific points of reflections. The proposed system can have applications in situations where the person can not stay in front of sensors or remain immobile for long time. On the other hand, some patients wear the non-invasive ventilatory masks to help with breathing. This mask prevents the remote respiratory monitoring system to operate, because the system measures the airflow of nasal or oral breathing. The proposed system can be of potential use for this situation.

A preliminary evaluation of the proposed system was done before [26]. In this paper, the longer data from the proposed ultrasound system in different times are collected. PPG sensor and spirometer as two main and most common methods for heart and breath cycles monitoring are selected to evaluate and validate the performance of the system. It is to be noted that the data is collected for a healthy subject only to do the first-level system validation compared to the above mentioned state-of-art studies.

\section{PhysicAl PRinciples AND Ultrasound WAVE GENERATION}

The sensor node is a piezo transducer mounted by epoxy on a plastic surface. The plastic surface acts as an acoustic matching layer. Only a conductive soft material, such as ultrasound gel, is needed to be be rubbed on the skin surface to remove air gaps between the sensor and skin. There are many alternatives, such as water, baby oil or hand cream which have almost equal performance. So, the patient is not limited to use the ultrasound gel. The proposed system lessens the number of connected electrodes or sensors directly on the skin. Unlike ECG leads, the proposed system does not need adhesive (adhesive may cause irritations on sensitive skins , infants or injured bodies) to attach the sensor node on skin. One strap band is enough to hold the sensory head on its position and maintain a constant light pressure on the sensor to the skin. Then the conductive medium enables a tight bond between the skin and the probe or transducer, letting wave transmissions directly to the tissues underneath.

In soft tissues, about $80 \%$ of the ultrasound wave is absorbed by the tissue resulting in local heat production on 
cells. Attenuation coefficients and acoustic impedances of some specific tissue types and mediums [27] are listed in Table I. Higher value of attenuation coefficient means more

TABLE I: Acoustic Properties of Biological Tissues

\begin{tabular}{|c|c|c|}
\hline Medium & $\begin{array}{c}\text { Attenuation Coefficient } \\
(\mathrm{dB} / \mathrm{cm})\end{array}$ & $\begin{array}{c}\text { Acoustic Impedance } \\
\left(\mathrm{kg} / \mathrm{m}^{2} . \mathrm{s} \text { or rayl }\right) * 10^{6}\end{array}$ \\
\hline Water & 0.002 & 1.48 \\
\hline Blood & 0.18 & 1.61 \\
\hline Fat & 0.63 & 1.38 \\
\hline Muscle & $1.3-3.3$ & 1.62 \\
\hline Bone & 5.0 & 6.0 \\
\hline
\end{tabular}

attenuated ultrasound wave passes the medium. For instance, bones with a very high attenuation coefficient hardly allows beam transmission through them. In addition, due to the high acoustic impedance mismatch between bone and tissues, there is a high intensity beam reflection. For small upper body motions, the ultrasound waves emitted by the transducer can still pass through the rib cage bones gap while a big skin movement may results a noticeable sensor displacement, causing the ultrasound wave not to pass through the rib cage bones gap. This misplacement leads to an error in reading ultrasound reflection. Such a big displacements rarely happen when the patient is not in intense motion.

In the pulsed ultrasound method, electrical pulses applied repetitively at a certain rate are called transmitted beams. In this design, the Pulse Repetition Frequency (PRF) equals 50 $\mathrm{Hz}$ and pulse duration $\tau \approx 5 \mu$ s when 5 pulses are applied on piezo sensors. So, the Duty Factor $D F=\tau / P R F=2.5 \times$ $10^{-4}$. Acoustic pressure and instantaneous acoustic intensity can be found from equation 1. Here, the $\rho . c$ is also called the acoustic impedance of the medium.

$$
I=\frac{p^{2}}{\rho . c} \quad\left[W / \mathrm{cm}^{2}\right] \quad p=\frac{V}{M(f)} \quad\left[P_{a}\right]
$$

$\mathrm{p}=$ Instantaneous acoustic pressure

$\rho=$ Density of the medium $\left(K g / \mathrm{m}^{3}\right)$

$\mathrm{c}=$ Speed of sound in the medium $(\mathrm{m} / \mathrm{s})$

$\mathbf{M}=$ Sensitivity of sensor $\left(V / P_{a}\right)$

To address the safety of designed ultrasound system, intensity limits provided by the Food and Drug Administration (FDA) agency [28] should be considered. Two known intensities are measured to ensure that their level is below the limits assigned by the FDA. The system is capable to stimulate the transducer with maximum $\pm 20 \mathrm{~V}$ differential pulses. When a maximum $40 V_{P P}( \pm 20 \mathrm{~V}$ differential $)$ voltage is applied on the transducer, the Spatial Peak Temporal Average intensity $I_{S P T A}=I_{S P P A} \times D F$ is equal to $15.4 \mathrm{~mW} / \mathrm{cm}^{2}$ where the Spatial Peak Pulse Average intensity is $I_{S P P A}=I / P D=61.7\left(W / \mathrm{cm}^{2}\right)$. PD is the pulse duration when the pulse pressure reaches $10 \%$ and $90 \%$ of its maximum value. The parameters are found smaller than the maximum intensities by FDA, being $I_{S P P A}=190 \mathrm{~W} / \mathrm{cm}^{2}$ and $I_{S P T A}=430 \mathrm{~mW} / \mathrm{cm}^{2}$. According to the Eq. 1, the ultrasound wave intensity is proportional to the applied voltage level with power of two.
Vibration (resonant) modes of piezo-ceramics depend on their shape, polarization orientation and the direction of the electric field. Each of these modes has unique resonant frequencies and characteristics. The axial resolution of ultrasound waves in a $1 \mathrm{MHz}$ piezo disks, or the average accuracy of the sound waves, can be calculated as below (considering the average sound velocity in tissue $c=1540 \mathrm{~m} / \mathrm{s})$ :

Axial Resolution:

$$
\theta_{z}=\frac{\lambda}{2}=\frac{c}{2 f}=\frac{1540(\mathrm{~m} / \mathrm{s})}{2 * 10^{6}} \simeq 0.77 \mathrm{~mm}
$$

The piezo sound waves accuracy of $0.77 \mathrm{~mm}$ suffices to measure the regular heart motions, as well as its movements due to the breathing cycles. Although piezo sensor's operation at higher frequencies improves the accuracy of the measurements, but the power consumption and system complexity increase accordingly, which is not beneficial for a round-aclock or a wearable embedded monitoring system.

\section{A. Pulse Generation and Observation}

As discussed in the previous section, the average intensity of pulses depends on pulse repetition factor and the voltage level. Conventional medical ultrasound systems stimulate piezo sensors by high voltages (approximately 100V) to increase the wave intensity, resulting higher amplitude of echoes from deeper organs, while maintaining the limitations assigned by FDA. In this study the goal is not detecting the movements of far tissues or even farther than heart left chamber. Practical experiments have shown that low energy acoustic pulses are enough to track the tissue, meaning a lower voltage pulse is sufficient to stimulate the transducer. In this study, we applied $\pm 8 \mathrm{~V}$ differential pulses on transducer. Initial observations have shown that this voltage rating is sufficient for heart motion tracking. Monitoring for subjects with thicker skin and rib cage may need higher acoustic energies as the depth of penetration increases. Lowering the voltage not only decreases the intensity of pulses, which is proportional with the degree of two in Eq.1, but also lessens the system power consumption. Although the change in transducer stimulation voltage from $\pm 20 \mathrm{~V}$ to $\pm 8 \mathrm{~V}$ results less than $2 \mathrm{~mA}$ current consumption changes in this design, this amount would be considerable in a longer time power consumption. In addition, it is better to lessen the ultrasound wave intensities and inappreciable side effects on the body. However, ultrasound is known for an excellent safety record.

In ultrasound B and M Mode imaging, for every transmitted beam, there is a reflection carrying information on its frequency, phase, amplitude and the time of flight $(\mathrm{ToF})$ of peaks. As the repetition factor increases, the refreshment of these information increases as well, whilst the $I_{S P P A}$ rises proportionally which is not favorable. A trade-off between these two parameters resulted the PRF $=50 \mathrm{~Hz}$ to achieve a suitable accuracy of $2 \%$ in reading every single record from reflected signals.

Fig. 1 shows an example of received waveforms when the sensor is placed on the chest. The orange wave is the envelope 


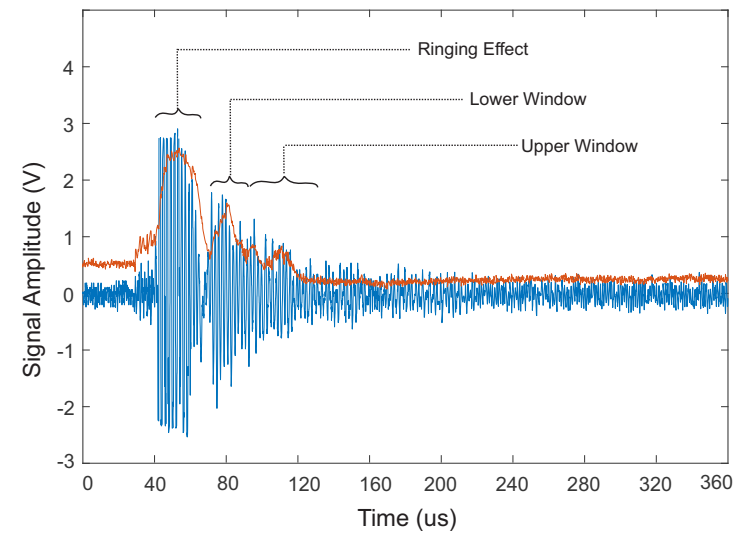

Fig. 1: An example of amplified received (Rx) ultrasound waveform from transmitted beams $(\mathrm{Tx})$ and its extracted envelop. 200 samples taken at $700 \mathrm{Ks} / \mathrm{s}$, called a Record.

of echoes received. The required information lies under the peaks amplitude and their locations (ToF), explained more in Section III. The biggest peaks marked on this figure are the result of sensors ringing effect. A low impedance electrical load for a short period is applied to diminish the ringing effect once the pulse generation ends.

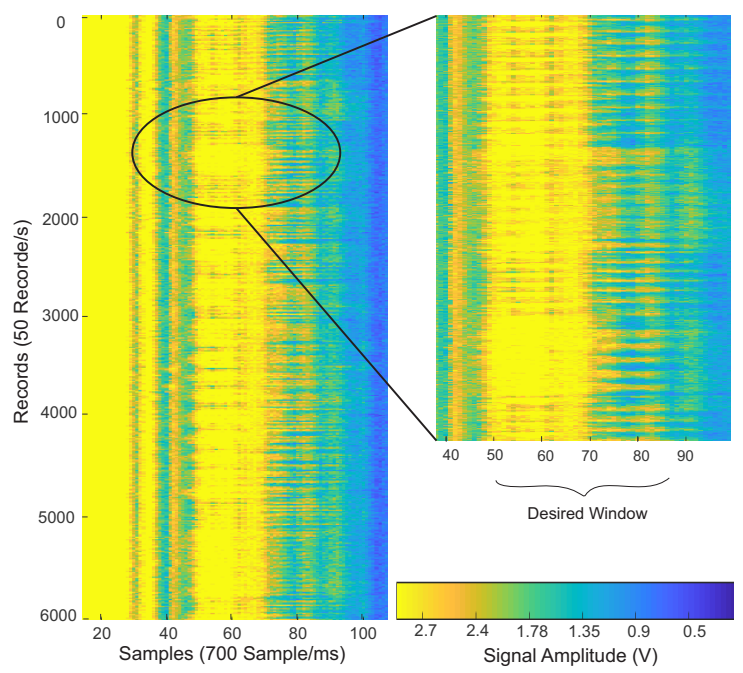

Fig. 2: 6000 records taken at every $20 \mathrm{~ms}$. A data for a subject in rest with a zoomed area for better visualization.

Fig. 2 depicts a two minutes data recorded for a subject in rest without body movements to monitor the heart motions. This 6000 records is a series of the envelope signals, such as one shown in Fig. 1, recorded at every 20 ms. Results are similar to M-Mode ultrasound imaging where $\mathrm{M}$ stands for motion tracking over time. In this image, the two main systolic and diastolic heart cycles are evident more in the zoomed area in Fig. 2. The narrow yellow lines within samples 70 to 85 indicate the systolic cycles and samples 50 to 70 the diastolic.

\section{System ARChiteCtURE}

Fig. 3 is the overall block diagram of the system designed and implemented to extract, monitor and record the ultrasound data and validate them with references. The ultrasound system consists of a semi high voltage stimulator and the receiver path to detect, magnify and digitize reflected echoes. The FPGA controls all blocks through digital pulses. It communicates serially to a computer for data collection and initialization. Electrical pulses are applied on a piezo transducer mounted by an epoxy on a thin hard plastic surface. The thickness of the plastic layer is chosen to be $\lambda / 4$ to have the least reflections from this matching layer.

In order to increase the sense of reflections from deeper tissue levels, it is required to improve the signal to noise ratio (SNR). Increasing the amplifier gain of the receiver circuit does not improve the SNR significantly due to many noise resources such as sensor ringing effect, component and power supply noises. Moreover, it magnifies only low voltage electrical signals from the transducer. There is a need to increase the amplitude of reflected acoustic waves. The simplest way is an increase in energy of transmitted acoustic waves, while maintaining FDA limits as discussed before, to obtain higher magnitude of reflections. Hence, a High Voltage (HV) Pulser is used to generate a differential pulses up to \pm 20 volt to increase the intensity of ultrasound waves. An IC MAX-14808 performs as the front-end high voltage (HV) pulser, switch and damper in a single chip. A Cyclone V FPGA applies digital control pulses to the HV-pulser. This IC has embedded independent power supplies and level shifters that allow signal transmission without the external HV capacitors. It has integrated grass-clipping diodes to isolate the receive (Rx) path from high voltage spikes on the transmit (Tx) path. The IC operates in Three-Level mode for burst pulse generation, shown in Fig. 3, controlled by the two square pulses shown in this figure. It features an on-resistance $500 \Omega$ damping circuit to discharge the pulser's output internal node as soon as the transmit burst is over. Two controller signals from the FPGA control the operation into the Transmit Disable mode. In this mode, the T/R switch is ON and the sensor's output will be directed to the RX path while the damper is ON. The burst transmission period lasts 5 pulses, which is 5 us. In addition to the damping circuit, once the pulse generation is over, a zero voltage will be applied on the piezo transducer, making a short circuit for less than 2 us, to decrease the impact of unwanted signals due to the sensors ringing effect.

A two-stage linear amplifier with a wide passive bandpass filter magnifies reflected ultrasound beams from undesired high and low frequency components of the signal. The magnified signal is passed to an envelope detector and a Dynamic Average Threshold Crossing (D-ATC) block [29], which helps to reduce the digital signal processing effort by finding indexes of the sampled signals where the amplitude passes a threshold value in each record [30]. It takes samples of the enveloped signal at the same frequency as the carrier signal $(1 \mathrm{MHz})$, and the result of every sampled signal is a binary value. Each record stores an array of these logical values. Consecutive data of these records reveal the location of the signal amplitude's variations when a new record is compared with the latest one. This technique automates the calculation by finding the centerpoint of these variations to find the desired window. Fig. 2 shows an experimental dataset, where the center is sample 


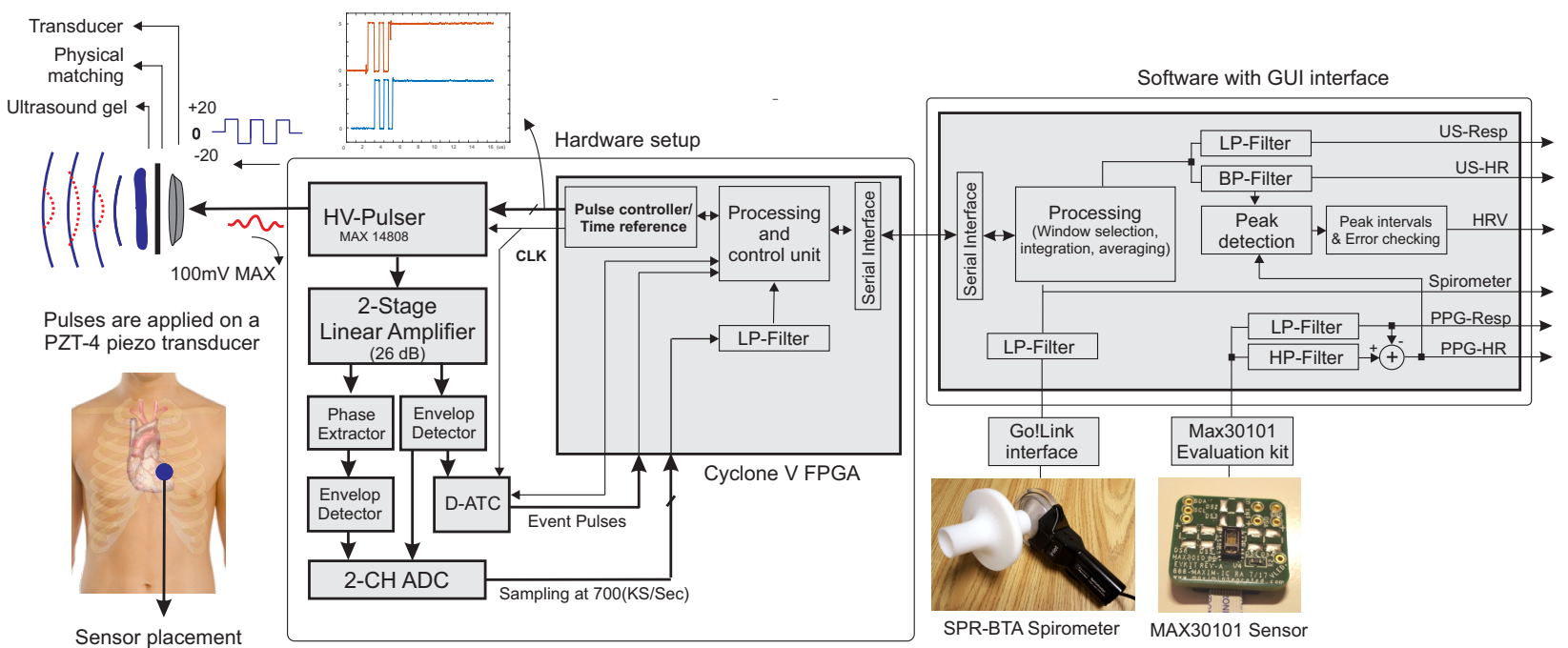

Fig. 3: Ultrasound system designed to continuously measure and monitor heart and respiratory cycles. Spirometer and PPG sensors are used as references for ultrasound data validation.

number 70 and the amplitude of the signal in each record varies within the desired window, period of sample numbers 50 to 90 . The window's center index varies in different conditions such as body position or the thickness of chest tissues in different subjects. Based on observations, breathing impacts a negligible shift in the center point.

In shallow respirations, the peak location variations of the envelop signal are not evident due to the low axial resolution of the sensor and reflected waves. We observed that these variations are more evident in the phase of the reflections. Therefore, a phase shift comparator can relate the phase of the transmitted signals to the received echoes. This shift can be extracted by a simple analog switch. An analog multiplexer/demultiplexer with logic-level conversion, CD4053 from Texas Instruments, is used here. Logical voltage level of a square $1 \mathrm{MHz}$ pulses handles the control of switch to divide its input signal (amplified received signals shown in Fig. 1) into two phases. Accordingly, the portion of the signal having different phase than the carrier is revealed. Due to the switch leakages, instability in the phase of reflections, TX pulse jitters and system noise, the output is filtered by an envelop detector to filter the high frequency noises and spikes to extract the envelop of the signals are out of phase.

A 2-channel, 8-bit resolution, fast analog to digital converter (ADC) with the sampling frequency of $700 \mathrm{Ks} / \mathrm{s}$ converts the two analog signals once the system switches to RX mode. For each record, 200 samples are taken from the signal, which lasts about 285 usec. This duration is the maximum time where echoes are observable in terms of the peak intensities and depth of the desired observation. It is worth noting that the energy of ultrasound waves attenuates as it moves through tissues. The amplitude decreases approximately by $1 \mathrm{~dB}$ per $1 \mathrm{MHz}$ per 1 centimeter traveled. So, our ultrasound wave weakens by $1 \mathrm{~dB}$ for each centimeter of penetration.

By using the analog multiplexer as a phase extractor, the D-ATC block and envelope detector, we reduce the workload of the DSP unit. Although digital processing has other advantages, to apply the above mentioned processing and detections in digital, it requires a fast analog to digital converter with a sampling frequency of at least $2 \mathrm{MHz}$ (by Nyquist sampling theorem) to reconstruct the original signal. In this case, to quantize the shift in phase in digital side with a good accuracy, the necessity for a faster than 2 Msamples/sec ADC increases. In addition, a sophisticated processing unit and ADC should be utilized to overcome the requirements for the information extraction. The data is logged and processed by MATLAB (Matrix Laboratory, USA) with a user-friendly GUI interface. The processing on ultrasound data could be done on FPGA. However, in order to make a better timing comparison between all three resources, it is done on a computer.

Fig. 2 shows an example of 6000 records taken at every 20 ms (120 seconds in total), while the subject starts with two breath-holds (with full inhalation and exhalation), then four slow breaths followed by eight fast breaths and ended with two other breath-hold. In this figure, the amplitude variations of each record over the time contains the information on the heart and breathing rates. The $\mathrm{X}$ axis is Samples (taken at 700 $\mathrm{KS} / \mathrm{Sec}$ ) and $\mathrm{Y}$ axis is the sampling repetition or Records of 50 Records/sec. The integral of the signal or the mean value within the desired window (discussed in the last section) of each record gives a value $\left(M_{j}\right)$.

$$
M_{j}=\frac{1}{U B-L B} \sum_{i=L B}^{U B} S_{j i}
$$

where $\mathrm{j}$ and $\mathrm{i}$ are the record and sample indexes respectively (on axises $\mathrm{Y}$ and $\mathrm{X}$ accordingly). The LB and UB are two lower and upper bounds of the desired window using data of the D-ATC block. A series of $M_{j}$ values for all records produces a signal shown in Fig. 4A. Two low-pass and highpass FIR filters, with cut-off frequency of approximately 0.5 $\mathrm{Hz}$ extract the low frequency element of the raw signal, which 


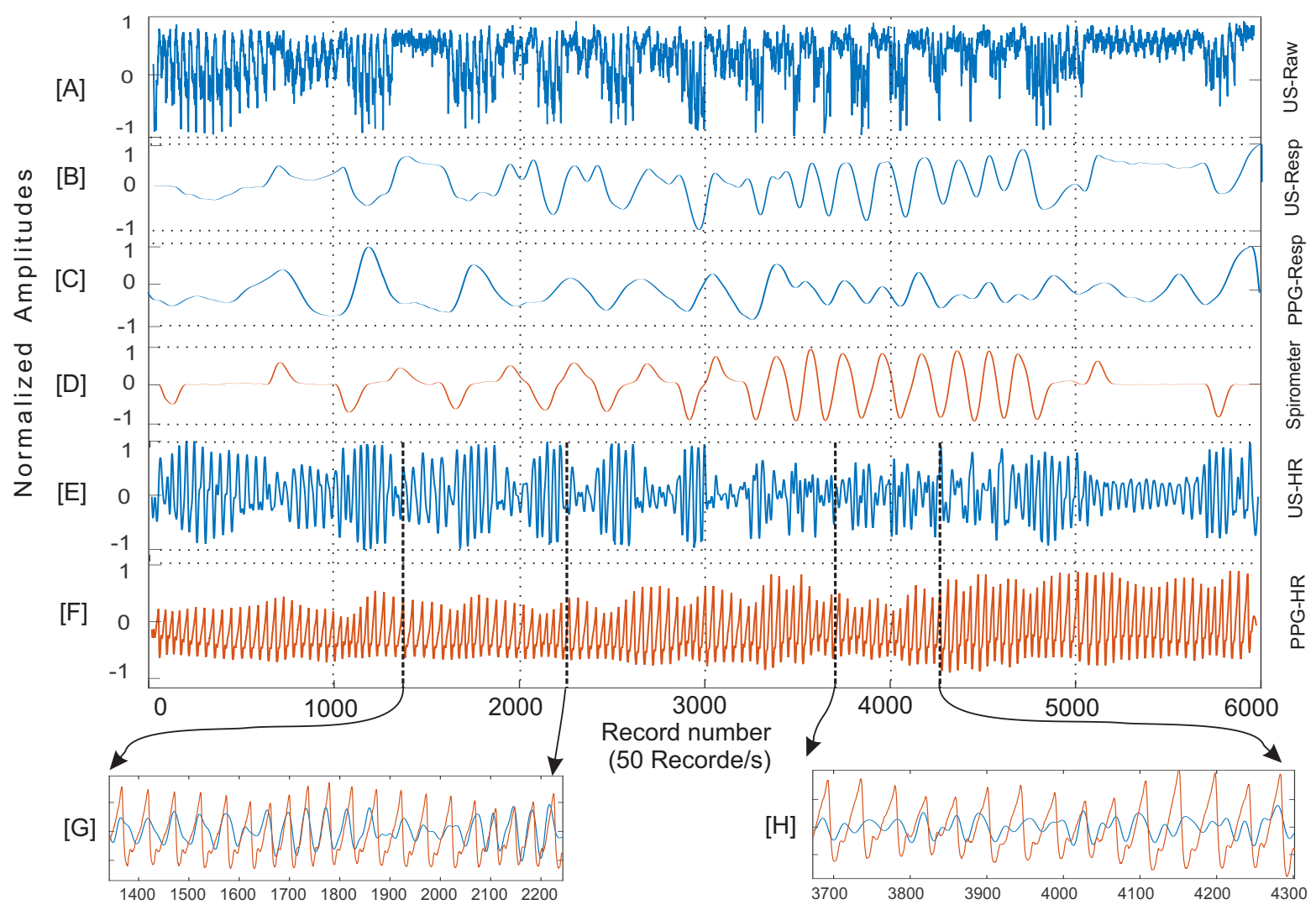

Fig. 4: (A)Raw data resulted from a series of mean values for each record within the desired window in Fig. 2. (B)Low frequency component of the signal (A) shows breathing cycles. (C)low-passed signal of the PPG sensor as its respiratory waveform. (D)Spirometry signal as a reference to measure the airflow during respiration cycles. (E)High frequency component of the signal (A) represents heart rate (HR) signal. (F) Pulse oximetry heart rate signal as a reference for average heart beats and its variation (HRV) verification. $(\mathrm{G})$ and $(\mathrm{H})$ are zoomed area for a better representation of US-HR waveform versus PPG-HR and the impact of breathing on US-HR waveform.

is the respiration trend (Fig. 4B), and the high frequency elements related to the heart cycles (Fig. 4D). High order FIR filters are used to filter because of linearity in phase which is a very important criterion for filter selection, especially for heart rate data.

\section{Data Analysis AND Experimental Results}

\section{A. Ultrasound Data Validation}

Six healthy subjects with ages of 26 to 34 were asked to do the test. We applied two tests on each subject prolonged two minutes each, and the average of the two tests are reported in the table II. Fig.4 depicts one out of two data sets of the first subject.

To validate the data obtained from ultrasound system, two references from which the breathing and heart rate can be calculated accurately are needed. Spirometers, pulse oximetry sensors (PPG) and electrocardiogram (ECG) are most known clinical and commercial references. Therefore, a SPR-BTA spirometer with GO!Link data logger is used to measure oral breathing in rest condition. A nose clip is used to prevent nasal breathing. This device measures the amount of airflow not the volume. Accordingly, the signal level returns to zero in breath holds as shown in Fig. 4D.
As a real-time reference for the heart rate tracking, we used MAX30101 evaluation kit which provides a proven design to evaluate the integrated pulse-oximetry and heart rate monitoring integrated circuit of MAX30101 sensor. PPG sensor is selected as a comparative technique to the proposed ultrasound system. Some studies proposed the use of PPG sensors for respiration estimation as discussed in the Introduction.

\section{B. Respiratory signal validation}

In this study we used two low-pass and high-pass filters to extract the heart and respiratory cycles from the PPG sensor's data and compared its respiratory waveform with the spirometer to validate the reliability of this sensor as a respiratory monitoring.

Fig. 4B is the obtained ultrasound respiratory (US-Resp) waveform from the proposed system for a 2 minutes record. The first minute is a normal breathing started with two long breath-holds. It begins with a full inhalation and a full exhalation ends at 12 seconds. Note that only the first rising or falling edge of respiratory waveform for each cycle in Fig. 4D should be matched with Fig. 4B, because the spirometer is a flow-meter sensor not air volume. Moreover, the output of phase detector is added to the low frequency component of 
US-Resp for better sense of respiration. Except some minor delays at these edges such as around the record number 2000, the US-Resp shows a high correlation with spirometer. The second half of the waveform shows a very close agreement in deep and fast breathing with average breathing length of 3.5 second and ended with two long hold-breaths. In a study [31], they measured the diaphragmatic and cardiac motion during breath holds. They found an average velocity of $0.15 \mathrm{~mm} / \mathrm{sec}$ of diaphragm at end expiration, which applies a pressure on the cardiopulmonary system. The impact of this motion is observed in the US-Resp waveform distinctly. For example, around record number 1300 in Fig. 4B where the subject had a full exhalation and suspension on his breath, the signal level lowers gradually, same as records around 5100.

Based on observations on 12 experiments of the 6 subjects, a weak correlation between the PPG and spirometer waveforms are found. According to the discussion on indirect methods for respiratory monitoring systems in the introduction, the PPG signal level has a late response to the breathing. In fast breathing, this response is evident when compared to a reference such as spirometer and the proposed ultrasound system, as plotted in Fig. 4C on records 3000 to 5000. The Fig. 4C is the low-pass filtered waveform of the PPG sensor's data. It is evident in this figure that the signal contains some peaks and valleys where there is no breathing activity, such as the records 200 to 800 and 5000 to 6000 .

In this study, subjects are asked to follow a similar breathing pattern as in Fig.4. Respiratory waveforms are statically analyzed using the following equations to find the sensitivity and specificity of the PPG and proposed sensor in reference to our gold standard.

$$
\text { Sensitivity }=\frac{T P}{T P+F N}
$$

In this equation, the true positive (TP) is the number of correctly detected full breathing operations and false negative (FN) is the number of breaths wrongly classified as negative. True negative (TN) means the time both the gold standard (spirometer) and the sensor do not detect any breathing.

$$
\text { Specificity }=\frac{T N}{T N+F P}
$$

For patients having sleep apnea, it is important to avoid false detection, which is the time the patient is suffocated but the sensor is showing a breathing operation. An example of this condition is evident in Fig. 4C from records 5000 to 6000. The PPG sensor shows an inhalation whilst the subject was holding his breath. Therefore, the False Positive (FP) is an important parameter in respiratory monitoring to be minimum, meaning a higher value of specificity.

\section{Heart Rate validation and assessment}

Fig. 4E shows the ultrasound heart rate waveform (US$\mathrm{HR}$ ) and can be compared with the reference signal in the time domain in Fig. 4F. The average heart rate measured by ultrasound sensor is 64.4 BPM and 68.7 BPM for PPG sensor, resulting $93.8 \%$ accuracy for the two minutes data in this figure. Two periods of these two waveforms are zoomed in Fig. $4 \mathrm{H}$ and $\mathrm{G}$ for better understanding of the signal shape, amplitude and phase which is discussed in details in next section.

To estimate the peak to peak time intervals of heart beats, peak extraction and zero crossings are two common techniques. In this application, the signal offset is subject to fluctuations due to reasons such as sensor displacement, body motions and scattered ultrasound waves from surrounded organs. So, peak to peak (PP) detectors performs more reliable and accurate time interval estimation. The PP time intervals of heart rate signals are extracted to validate the heart rate variability (HRV) of ultrasound system. Although the PPG sensor provides pulse rate variability (PRV), not HRV, there is a significant correlation between these two values when compared with ECG signal [32]. The heart rate signal of this sensor is shown in Fig. 4F.

Since the heart rate detection is based on the heart motions, any sort of external pressure to the pericardium sac can apply displacement to the heart, leading longer or shorter PP detection. Breathing is one of the known sources causing this error as shown in Fig. $4 \mathrm{H}$ where the heart rate signal has some unwanted or misaligned peaks compared to the reference which is the PPG signal. The error increases as the speed and intensity of breathing increase. Fig. 4G shows a very well matching compared to $4 \mathrm{H}$. There is a minor error in detected PP time around sample number 2070 which is a result of inhalation occurring at the same time as heart beat. Therefore, an error detector is added to the system to replace the detected PP time interval with the previous one if this time is higher than $30 \%$ of an average within past three PP intervals. On the other hand, skip a PP interval if its duration is less than $70 \%$ of the average. This error for each subject is listed in the Table II.

Observations and results have shown higher error in heart beat detection and more conformal trend in respiration at faster and deeper breathing. However, a very high correlation between the ultrasound signals and references indicates that the proposed method is promising to be used in a human health tracking systems specially for real-time monitoring of breathing disorder and heart rate variation abnormality during sleep.

PP time intervals of ultrasound and PPG sensors are shown in Fig 5. The US-HR presents the same trend as PPG-HR except heart beats numbers 90 to 100 with a little error. This mismatch is a result of fast breathing explained before. For the whole two minutes, 131 beats are detected which matches with the average time of the whole PP intervals. In addition, the respiratory sinus arrhythmia biofeedback (RSA) is evident in Fig. 5 when compared to any respiratory waveforms in Fig. 4. The beats time intervals decreases during inhalation, such as the inspiration at sample 1000 in Fig. 4B corresponds reduction in time around beats number 20 in Fig. 5 .

Box plot of the obtained PP intervals for both PPG and US sensor for all five tests are summarized in Fig. 6. This figure shows the dispersion of intervals versus the mid point which is a median for each data set. 
TABLE II: A summary of experimental test results on five subjects.

\begin{tabular}{|c|c|c|c|c|c|c|c|c|c|}
\hline \multirow{3}{*}{ Subject } & \multicolumn{4}{|c|}{ Heart rate } & \multicolumn{5}{|c|}{ Respiratory rate } \\
\hline & \multirow{2}{*}{$\begin{array}{c}\text { US } \\
(\mathrm{BPM})\end{array}$} & \multirow{2}{*}{$\begin{array}{c}\text { PPG } \\
(\mathrm{BPM})\end{array}$} & \multirow{2}{*}{$\begin{array}{l}\text { Error } \\
(\mathrm{BPM})\end{array}$} & \multirow{2}{*}{$\begin{array}{l}\text { Error in detecting } \\
\text { true heart beats }\end{array}$} & \multirow{2}{*}{$\begin{array}{l}\text { Breaths } \\
\text { (per min) }\end{array}$} & \multicolumn{2}{|c|}{ Sensitivity } & \multicolumn{2}{|c|}{ Specificity } \\
\hline & & & & & & US & PPG & US & PPG \\
\hline 1 & 66.34 & 67.5 & $2.78 \%$ & $9.10 \%$ & 7.5 & $100 \%$ & $88.20 \%$ & $88.90 \%$ & $84.20 \%$ \\
\hline 2 & 72.35 & 78 & $7.20 \%$ & $14.20 \%$ & 10 & $89.10 \%$ & $80.30 \%$ & $91.80 \%$ & $83.70 \%$ \\
\hline 3 & 72.25 & 72.2 & $0.07 \%$ & $9.02 \%$ & 9 & $94.10 \%$ & $84.20 \%$ & $94.10 \%$ & $94.40 \%$ \\
\hline 4 & 61.7 & 62.15 & $0.67 \%$ & $12.70 \%$ & 16 & $87.80 \%$ & $57.50 \%$ & $98.70 \%$ & $82.50 \%$ \\
\hline 5 & 74.3 & 79.8 & $6.90 \%$ & $18.10 \%$ & 15 & $98.30 \%$ & $38.50 \%$ & $95.20 \%$ & $55.20 \%$ \\
\hline 6 & 84.9 & 83.2 & $2.05 \%$ & $9.03 \%$ & 20 & $97.40 \%$ & $65 \%$ & $95.20 \%$ & $74.50 \%$ \\
\hline Average & 71.9 & 73.8 & $3.30 \%$ & $12.00 \%$ & 12.9 & $94.50 \%$ & $69.00 \%$ & $94.00 \%$ & $79.10 \%$ \\
\hline
\end{tabular}

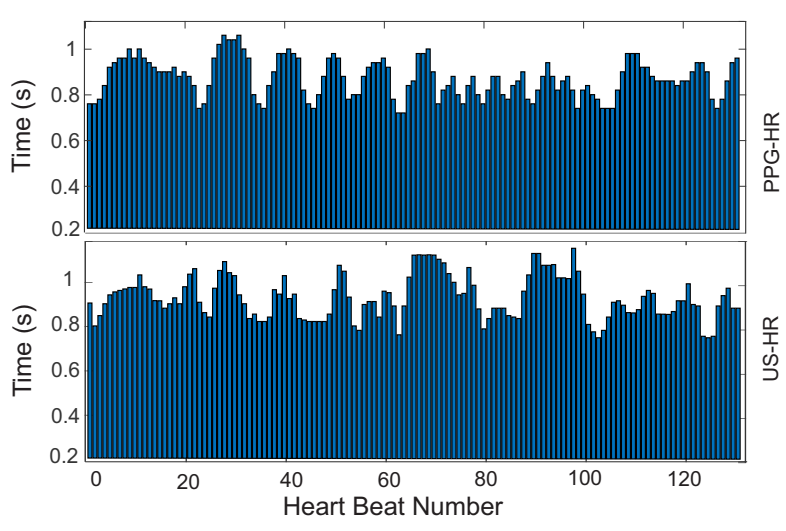

Fig. 5: Peak to peak time intervals of Fig. 4E and $F$ show heart rate variability (HRV) for ultrasound and PPG sensors visualizing 131 beats.

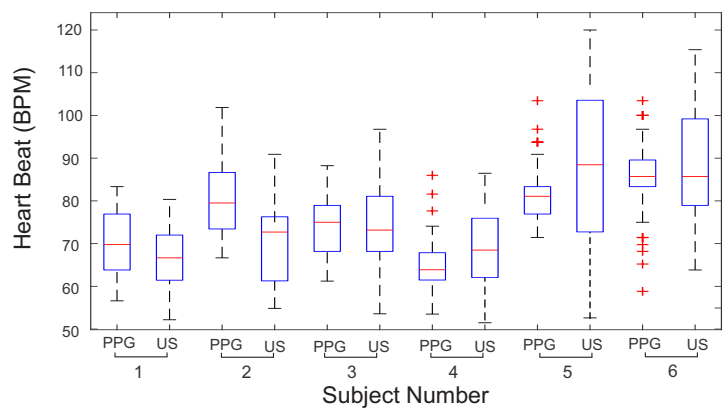

Fig. 6: Statistical visualization of peak to peak (PP) time intervals of PPG and ultrasound (US) sensors for six subjects.

\section{Hardware resources and noise analysis}

The proposed system requires +3.3 voltage sources for the digital and analog integrated circuits and the differential \pm 5 to \pm 20 volts from an external DC linear power supply for the transducer stimulation. It consumes $34 \mathrm{~mA}$ for the digital and analog circuit. The transducer itself consumes less than $0.1 \mathrm{~mA}$ on different voltages. Only 393 logical blocks of a Cyclone II FPGA are used. The average SNR values for HR and RESP signals measured 2 and $3.2 \mathrm{~dB}$, respectively, from the following equations:

$$
P_{n}=\frac{1}{N} \sum_{j=1}^{N \_ \text {Records }} M_{j}^{2}
$$

where $M_{j}$ is the mean value in Eq.3 and the $P_{n}$ is the power of signal when it does not contain any useful signal (the sensor is not placed on body). The same equation as 6 is used to find the $P_{s}$, the power of signal+noise mixture, when the sensor is placed on the body and system is measuring heart or respiratory cycles. Finally, the SNR could be calculated by:

$$
S N R=10 \log _{10} \frac{P_{s}-P_{n}}{P_{n}}
$$

TABLE III: The designed hardware parameters summary

\begin{tabular}{|l|l|c|}
\hline Parameter & Value & Unit \\
\hline SNR(RESP) & 3.25 & $\mathrm{~dB}$ \\
\hline SNR(HR) & 2.1 & $\mathrm{~dB}$ \\
\hline Amplifier gain & 20 & $\mathrm{~dB}$ \\
\hline Total current & 34 or less & $\mathrm{mA}$ \\
\hline Sensor current & 0.1 or less & $\mathrm{mA}$ \\
\hline Voltage ratings & +3.3 and \pm 10 & $\mathrm{~V}$ \\
\hline FPGA Logics & 393 & - \\
\hline
\end{tabular}

Skin motions can cause the movements of the sensor position. Large movements can induce the system failure to observe organ motions as the rib cage bones will block the ultrasound path from the transducer to the organ. However, the proposed system would not generate false positive detections even if the rib cage bones block the ultrasound path. An array of three to five sensors seating next to each other would help for better motion detection. Assuming a 0.5 to 1 centimeter space between each sensor guarantees that at least one transducer will observe motions, even if others are blocked by rib cages. As explained before about the desired window, our system looks into motions observed within this period of time. Any other noise, such as skin surface reflection, ringing effect and acoustic mismatch have the most amplitude on the signal within the period of 0 to 40 samples in Fig. 2, and least amplitude after the 40 samples which is our desired period. Therefore, the proposed system would be less affected by these other sources of noise.

\section{Discussion AND CONCLUSION}

In this study, a novel approach for long-term monitoring of heart and respiratory as the two main vital signs was proposed. The aim was to design a multi parameter monitoring system based on only one sensory node element and less wiring connectivity to the body. Derived waveforms from ultrasound signals carried two main information, higher frequency impulses resulted from the heart motions and lower 
frequencies of the signal due to the small respiratory motions of the heart and surrounded tissues. More investigation on the data revealed information correlated to the biofeedback of cardiopulmonary system, such as HRV, RSA and cardiac motion during suspended breathing due to the diaphragmatic pressures. Two clinical and commercial conventional methods for heart and respiratory monitoring systems are used to assess and validate the data obtained through the ultrasound system. The average accuracy of $96.7 \%$ is obtained for the heart beats per minute (BPM) measurements in reference to the PPG sensor for 6 healthy subjects. The respiratory waveform is also in good agreement with the waveform obtained from spirometer as a reference, having sensitivity and specificity of $94.5 \%$ and $94.0 \%$, respectively.

The system lacks a good physical and electrical impedance matchings. Improvements in this area can lead to a better SNR value and diminish the ringing effects. Our focus in the further studies will be integrating the system to be a wearable device and apply a comprehensive human test for validation on different subjects and body positions.

\section{REFERENCES}

[1] S. Patel, H. Park, P. Bonato, L. Chan, and M. Rodgers, "A review of wearable sensors and systems with application in rehabilitation," Journal of neuroengineering and rehabilitation, vol. 9, no. 1, p. 21, 2012.

[2] M. J. Lado, X. A. Vila, L. Rodríguez-Liñares, A. J. Méndez, D. N. Olivieri, and P. Félix, "Detecting sleep apnea by heart rate variability analysis: assessing the validity of databases and algorithms," Journal of medical systems, vol. 35, no. 4, pp. 473-481, 2011.

[3] G. Araujo, R. Freire, J. Silva, A. Oliveira, and E. Jaguaribe, "Breathing flow measurement with constant temperature hot-wire anemometer for forced oscillations technique," in Instrumentation and Measurement Technology Conference, 2004. IMTC 04. Proceedings of the 21st IEEE, vol. 1. IEEE, 2004, pp. 730-733.

[4] Y.-W. Bai, W.-T. Li, and Y.-W. Chen, "Design and implementation of an embedded monitor system for detection of a patient's breath by double webcams," in Medical Measurements and Applications Proceedings (MeMeA), 2010 IEEE International Workshop on. IEEE, 2010, pp. $171-176$.

[5] N. Bu, N. Ueno, and O. Fukuda, "Monitoring of respiration and heartbeat during sleep using a flexible piezoelectric film sensor and empirical mode decomposition," in 2007 29th Annual International Conference of the IEEE Engineering in Medicine and Biology Society, Aug 2007, pp. $1362-1366$.

[6] G. Loriga, N. Taccini, D. D. Rossi, and R. Paradiso, "Textile sensing interfaces for cardiopulmonary signs monitoring," in 2005 IEEE Engineering in Medicine and Biology 27th Annual Conference, Jan 2005, pp. 7349-7352.

[7] L.-G. Lindberg, H. Ugnell, and P. Öberg, "Monitoring of respiratory and heart rates using a fibre-optic sensor," Medical and Biological Engineering and Computing, vol. 30, no. 5, pp. 533-537, 1992.

[8] A. R. Fekr, K. Radecka, and Z. Zilic, "Design and evaluation of an intelligent remote tidal volume variability monitoring system in ehealth applications," IEEE journal of biomedical and health informatics, vol. 19, no. 5, pp. 1532-1548, 2015.

[9] S. D. Min, J. K. Kim, H. S. Shin, Y. H. Yun, C. K. Lee, and M. Lee, "Noncontact respiration rate measurement system using an ultrasonic proximity sensor," IEEE Sensors Journal, vol. 10, no. 11, pp. 1732 $1739,2010$.

[10] P. Arlotto, M. Grimaldi, R. Naeck, and J.-M. Ginoux, "An ultrasonic contactless sensor for breathing monitoring," Sensors, vol. 14, no. 8, p. 15371, 2014. [Online]. Available: http://www.mdpi.com/1424-8220/14/ $8 / 15371$

[11] S. Fouzas, K. N. Priftis, and M. B. Anthracopoulos, "Pulse oximetry in pediatric practice," Pediatrics, vol. 128, no. 4, pp. 740-752, 2011.

[12] R. Sahni, "Noninvasive monitoring by photoplethysmography," Clinics in perinatology, vol. 39, no. 3, pp. 573-583, 2012.
[13] S. A. Landsverk, L. O. Hoiseth, P. Kvandal, J. Hisdal, O. Skare, and K. A. Kirkeboen, "Poor agreement between respiratory variations in pulse oximetry photoplethysmographic waveform amplitude and pulse pressure in intensive care unit patients," Anesthesiology: The Journal of the American Society of Anesthesiologists, vol. 109, no. 5, pp. 849-855, 2008.

[14] K. H. Shelley, D. H. Jablonka, A. A. Awad, R. G. Stout, H. Rezkanna, and D. G. Silverman, "What is the best site for measuring the effect of ventilation on the pulse oximeter waveform?" Anesthesia \& Analgesia, vol. 103, no. 2, pp. 372-377, 2006.

[15] Y. Iyriboz, S. Powers, J. Morrow, D. Ayers, and G. Landry, "Accuracy of pulse oximeters in estimating heart rate at rest and during exercise." British journal of sports medicine, vol. 25, no. 3, pp. 162-164, 1991.

[16] A. Pantelopoulos and N. G. Bourbakis, "A survey on wearable sensorbased systems for health monitoring and prognosis," IEEE Transactions on Systems, Man, and Cybernetics, Part C (Applications and Reviews), vol. 40, no. 1, pp. 1-12, Jan 2010.

[17] F.-T. Wang, H.-L. Chan, C.-L. Wang, H.-M. Jian, and S.-H. Lin, "Instantaneous respiratory estimation from thoracic impedance by empirical mode decomposition," Sensors, vol. 15, no. 7, pp. 16372-16387, 2015.

[18] N. K. Kristiansen, J. Fleischer, M. Jensen, K. S. Andersen, and H. Nygaard, "Design and evaluation of a handheld impedance plethysmograph for measuring heart rate variability," Medical and Biological Engineering and Computing, vol. 43, no. 4, pp. 516-521, 2005.

[19] Z. A. Sani, A. Shalbaf, H. Behnam, and R. Shalbaf, "Automatic computation of left ventricular volume changes over a cardiac cycle from echocardiography images by nonlinear dimensionality reduction," Journal of digital imaging, vol. 28, no. 1, pp. 91-98, 2015.

[20] Y. Mehta and D. Arora, "Newer methods of cardiac output monitoring," World journal of cardiology, vol. 6, no. 9, p. 1022, 2014.

[21] T. Suwatanapongched, D. S. Gierada, R. M. Slone, T. K. Pilgram, and P. G. Tuteur, "Variation in diaphragm position and shape in adults with normal pulmonary function," CHEST Journal, vol. 123, no. 6, pp. 20192027, 2003.

[22] G. Shechter, C. Ozturk, J. R. Resar, and E. R. McVeigh, "Respiratory motion of the heart from free breathing coronary angiograms," IEEE transactions on medical imaging, vol. 23, no. 8, pp. 1046-1056, 2004.

[23] K. McLeish, D. L. Hill, D. Atkinson, J. M. Blackall, and R. Razavi, "A study of the motion and deformation of the heart due to respiration," IEEE transactions on medical imaging, vol. 21, no. 9, pp. 1142-1150, 2002.

[24] P. S. Rahko, "Evaluation of the skin-to-heart distance in the standing adult by two-dimensional echocardiography," Journal of the American Society of Echocardiography, vol. 21, no. 6, pp. 761-764, 2008.

[25] R. O'Rahilly and F. Müller, Basic human anatomy: a regional study of human structure. WB Saunders Company, 1983.

[26] A. Shahshahani, S. Bhadra, and Z. Zilic, "A continuous respiratory monitoring system using ultrasound piezo transducer," in Circuits and Systems (ISCAS), 2018 IEEE International Symposium on. IEEE, 2018, pp. 1-4.

[27] K. Shung, Diagnostic Ultrasound: Imaging and Blood Flow Measurements. Wiley, 1998. [Online]. Available: https://books.google.ca/books/ about/Medical_Instrumentation.html?id=fUlRAAAAMAAJ

[28] Food, D. Administration et al., "Guidance for industry and fda staff information for manufacturers seeking marketing clearance of diagnostic ultrasound systems and transducers," Silver Spring: US FDA, 2008.

[29] A. Shahshahani, D. R. Nafchi, and Z. Zilic, "Ultrasound sensors and its application in human heart rate monitoring," in 2017 IEEE International Symposium on Circuits and Systems (ISCAS), May 2017, pp. 1-4.

[30] A. Shahshahani, M. Shahshahani, P. M. Ros, A. Bonanno, M. Crepaldi, M. Martina, D. Demarchi, and G. Masera, "An all-digital spike-based ultra-low-power IR-UWB dynamic average threshold crossing scheme for muscle force wireless transmission," in Proceedings of the 2015 Design, Automation \& Test in Europe Conference \& Exhibition. EDA Consortium, 2015, pp. 1479-1484.

[31] A. E. Holland, J. W. Goldfarb, and R. R. Edelman, "Diaphragmatic and cardiac motion during suspended breathing: preliminary experience and implications for breath-hold $\mathrm{mr}$ imaging." Radiology, vol. 209, no. 2, pp. 483-489, 1998.

[32] R.-D. B. M. R.-T. M. M.-W. Robert Rauh, Robert Limley, "Comparison of heart rate variability and pulse rate variability detected with photoplethysmography," Proc.SPIE, vol. 5474, pp. $5474-5474$ - 12, 2004. [Online]. Available: http://dx.doi.org/10.1117/12.578377 